\title{
Crise du travail et redéfinition des frontières du social en Argentine à travers le prisme de la mobilisation des desocupados
}

Work crisis and the redefinition of social frontiers in Argentine: the mobilizations of the unemployed

Pía V. Rius

\section{(2) OpenEdition}

\section{Journals}

Édition électronique

URL : http://journals.openedition.org/travailemploi/6363

DOI : 10.4000/travailemploi.6363

ISSN : $1775-416 \mathrm{X}$

Éditeur

DARES - Ministère du Travail

Édition imprimée

Date de publication : 1 avril 2014

Pagination : $99-116$

ISSN : 0224-4365

\section{Référence électronique}

Pía V. Rius, "Crise du travail et redéfinition des frontières du social en Argentine à travers le prisme de la mobilisation des desocupados », Travail et Emploi [En ligne], 138 | avril-juin 2014, mis en ligne le 01 avril 2016, consulté le 08 mai 2019. URL : http://journals.openedition.org/travailemploi/6363 ; DOI : 10.4000/travailemploi.6363 


\title{
Crise du travail et redéfinition des frontières du social en Argentine à travers le prisme de la mobilisation des desocupados
}

\author{
Pía V. Rius(*)(1)
}

\begin{abstract}
En Argentine, les transformations de l'économie à l'œuvre depuis les années 1970 modifient la répartition des revenus, qui se dégrade encore durant les années 1990. La crise de la fin 2001, vécue comme hors du commun, témoigne des tensions entre les attentes d'intégration sociale par l'éducation et le travail et la politique néolibérale conduite par les gouvernements en place. À partir d'une analyse ethnographique et documentaire, l'article s'intéresse en particulier aux organisations de desocupados de la banlieue sud de Buenos Aires, à la manière dont les membres de ces collectifs et leurs proches vivent leurs conditions de travailleur et de chômeur. L'analyse des politiques sociales donne à voir une tension entre logique de droit au travail et logique de droit individuel à la protection sociale.
\end{abstract}

La crise économique que connaît actuellement la France apparaît avant tout comme une crise de la croissance et du chômage, qui fait suite à la crise financière de 2008-2009. En cela, elle peut constituer pour les chercheurs en sciences sociales une occasion de questionner la place du travail dans nos sociétés et invite à revenir sur les crises passées, comme celle qui a éclaté en Argentine à la fin de l'année 2001. Les conséquences sociales des années de récession économique (1998-2001) $\mathrm{y}$ ont été particulièrement importantes et se sont traduites par des transformations majeures du système politique, économique et social.

Cette crise signe l'échec du «modèle social argentin», qui reposait sur une monnaie stable au taux de change fixe avec le dollar américain. Elle témoigne également des tensions au sein de la société : alors que le pays se percevait comme accueillant, «ouvert à tous les hommes de bonne volonté(2) 》, depuis la fin des années 1990, l'augmentation du chômage et de la pauvreté, qui se traduit par des phénomènes d' «exclusion», participe à la transformation des rapports $\operatorname{sociaux}^{(3)}$.

(*) Centre lillois d'études et de recherches sociologiques et économiques (Clersé), Université Lille 1; piavrius@ehess.fr

(1) Je voudrais remercier Xavier Dunezat pour la lecture détaillée et généreuse d'une version préliminaire de cet article, qui m'a aidée à mieux le présenter ainsi qu'aux éditeurs de la revue pour leur soutien.

(2) Tels sont les termes du Préambule de la Constitution nationale que le président Raul Alfonsin a utilisés comme leitmotiv dans ses discours de campagne lors du retour à la démocratie à la fin de l'année 1983.

(3) Les débats autour des diverses formes de pauvreté urbaine nous invitent à rester prudent quant à l'usage du terme "inclusion» et son contraire, «exclusion» car on risque d'introduire un dualisme entre les deux (FASSIN, 1996), là où il y a plutôt une différenciation des formes d'inclusion.
En rendre compte à partir de la mobilisation des organisations des travailleurs desocupados (voir encadré) permet de saisir comment le chômage provoque une perte des repères, comment sont perçues les différentes mesures mises en œuvre (notamment les minima sociaux) et comment sont déterminées les populations et situations qui ont droit au secours de l'État. C'est au cours du processus de mobilisation que le chômage se construit et se définit en tant que problème public (4), désormais pris en compte en tant que tel dans la conception des politiques publiques. Ainsi, dans les années 1990, les mobilisations de la banlieue de Buenos Aires, marquée par les privatisations de plusieurs grandes entreprises publiques, par la désindustrialisation et la concentration de poches de pauvreté contribuent à mobiliser les gouvernements successifs.

L'Argentine des années 1990 a érigé en dogme indiscutable le «consensus de Washington», qui consiste en des politiques de libéralisation de l'économie et de flexibilisation du travail. Alors qu'en 1995 le chômage atteint un pic de $18 \%$ de la population active, le gouvernement de Carlos Menem, qui a mené ces politiques de réforme de l'État, est réélu. Si ces politiques néolibérales sont en cours depuis les années 1970, c'est la rapidité du creusement des inégalités de revenus et de patrimoine qui caractérise les années 1990. Dans ce contexte, les mobilisations de chômeurs et de sans-emploi tantôt

(4) La définition des problèmes publics, ici le chômage, n'est pas une simple conséquence de certaines conditions objectives. Elles découlent d'enjeux et questionnements qui cherchent à faire valoir leur légitimité. Suivant Erving Goffman (1991), Daniel CEFAİ $(1996,2011)$ considère que les problèmes publics sont thématisés «dans des cadres» et mobilisés dans des «opérations de cadrage». 


\section{Encadré Desocupados : chômeurs et sans emploi
en Argentine}

Le terme Movimiento de Trabajadores Desocupados (MTD) est la dénomination indigène que plusieurs collectifs se sont attribuée en Argentine à partir de 1996 et qui se traduit littéralement en français par "Mouvement de travailleurs au chômage». Néanmoins, nous préférons dans cet article conserver le terme desocupados car, dans le contexte institutionnel français, même si les représentations du "chômeur" sont fragmentées (DemAZIĖRE, 2003), les chômeurs ayant cotisé un certain temps, ont droit à une allocation-chômage pendant quelques mois (bien que ces droits viennent à s'épuiser) ce qui les rapproche de ceux qui en Argentine ont droit au "seguro de desempleo", soit environ $5 \%$ des actifs. En Argentine, la situation de la population désignée comme "sans emploi" est très instable en raison du poids du secteur informel et de la fragilité des entreprises. Les programmes de travail temporaire (PTT) sont accordés non seulement en situation de chômage - de courte ou longue durée et de tous âges confondus -, mais aussi en fonction des revenus du foyer, et donc du niveau de pauvreté. L'assurance chômage - seguro de desempleo destinée aux travailleurs ayant cotisé, ne couvre que $50,5 \%$ de la population active en 2003 et $55,5 \%$ en 2006 selon I'Indec (Insituto Nacional de Estadísticas y Censos).

Les mouvements de chômeurs argentins sont constitués par des organisations très labiles et aux registres d'action variables suivant les périodes. Contrairement à ce qui se passait lors des premiers piquetes ${ }^{(1)}$ en 1996 dans la province de Neuquén, la plupart des blocages à partir de 2004 durent seulement quelques heures. Exception notable à cette évolution générale: les blocages avec campement sur l'accès sud de Buenos Aires (Puente Pueyrredon) le 26 juin de chaque année - en mémoire de deux jeunes membres des mouvements de travailleurs desocupados (MTD) tués en 2002 par les forces de l'ordre - pour réclamer le jugement des responsables.

(1) Blocages de routes ou des ponts d'accès à la ville, cf. infra.

revendiquent un changement social profond, tantôt réclament une meilleure participation et inclusion de tous dans la société salariale. En réponse, les gouvernements successifs oscillent entre deux positions : prendre acte des revendications et entamer des processus de négociation; réprimer et traîner devant les tribunaux ceux qu'ils estiment être des fauteurs de troubles.

$\mathrm{Au}$ travers des mobilisations de chômeurs, nous nous intéressons ici plus particulièrement aux tensions entre logique de droit au travail et logique de droit individuel à la protection sociale, et à la manière dont les différents acteurs se les approprient collectivement. L'article s'appuie sur une enquête ethnographique, réalisée dans le cadre d'une thèse consacrée aux organisations de desocupados de la banlieue sud de Buenos Aires, menée au plus près de la vie quotidienne des habitants des quartiers pauvres (Rius, 2010). Nous avons effectué des observations entre juillet 2003 et août 2007 sur les stratégies économiques déployées par les habitants et leur participation civique et politique dans la vie quotidienne. Nous avons également réalisé des entretiens avec des membres des mouvements de desocupados et leurs proches, que nous avons complétés par une analyse documentaire ${ }^{(5)}$. Quelles sont les certitudes qui ont été ébranlées par l'expérience de la crise et du chômage, et quels sont les horizons communs qui peuvent alors se dessiner? En effet, être au chômage produit une dislocation telle que les repères jusqu'alors pertinents pour l'action ne sont plus valables. Alfred SchUTz (1987) explique ainsi que toute interprétation du monde est basée sur une «réserve d'expériences préalables», les nôtres propres, ou celles que nous ont transmises nos parents ou nos professeurs ; ces expériences, sous forme de «connaissances disponibles, fonctionnent comme schème de référence» (p. 12), schèmes qui peuvent s'effondrer au moment d'une crise.

Comment le travail est-il redéfini face à ces transformations? Quelles sont les situations qui justifient l'intervention de l'État ? Plus largement, peut-on parler - dans le cas argentin - d'un abandon de la «société salariale» (CASTEL, 1995) ? La multiplicité des régimes d'engagement a été étudiée au sein des classes moyennes, à partir de la dimension réflexive qui accompagne l'émergence des «nouveaux pauvres» (KessLer, 2002) ou dans le cadre des processus d'individuation des classes populaires à partir du passage de la figure du travailleur à celle du pauvre (MerkLEN, 2005). L'article présente des exemples, moins connus, d'engagement de desocupados, qui effectuent un travail sur eux-mêmes afin de compenser la perte de leurs repères dans un contexte de crise économique massive et ainsi maintenir leur dignité face à la crise du monde du travail.

Nous étudierons tout d'abord les évolutions du monde du travail qui ont structuré le processus de mobilisation des desocupados. La société salariale est l'horizon auquel aspirent la plupart des travailleurs, mais le «travail autonome» est aussi une référence largement partagée. Nous présenterons ensuite le traitement public du chômage ainsi que les programmes de travail temporaire (PTT) depuis 1998, à travers trois générations successives de programmes. Conçus comme un palliatif

(5) Nous avons travaillé sur différents rapports d'évaluation des politiques sociales concernées, commandités aussi bien par l'État que par les provinces ou des organismes bailleurs de fonds (Banque mondiale, Banque interaméricaine de développement) ainsi que sur des écrits produits par des organisations de desocupados. 
au chômage à destination des populations les plus démunies, ces programmes tentent d'articuler deux objectifs : la «lutte contre le chômage» et la «lutte contre la pauvreté». Enfin, nous verrons que les frontières du social ont été dessinées par l'intervention de l'État, qui tend à mettre en place une réponse d'inclusion sociale minimale par des politiques publiques d'activation des sans-emplois ${ }^{(6)}$, qui s'entrecroise avec des politiques familialistes.

\section{De la crise du travail à la mobilisation de desocupados}

Les mobilisations de desocupados ont émergé à la suite de transformations profondes et multidimensionnelles qui ont bouleversé les repères des travailleurs. Les entretiens avec des membres de ces organisations et avec leurs proches nous ont montré qu'il existe différentes manières d'appréhender à la fois de tels bouleversements et l'expérience militante. Ces dernières ne constituent en aucun cas une réponse mécanique aux transformations du rôle de l'Etat; elles permettent d'interpréter le processus de mobilisation au sein des organisations de desocupados comme une revendication qui se construit de manière relationnelle. Qu'il ait lieu avec des proches ou des autorités locales et nationales, le va-et-vient fait de confrontations, de négociations et de coopérations permet l'attribution des responsabilités et la définition des interventions possibles.

\section{Les transformations structurelles du monde du travail argentin}

Le processus d'industrialisation de l'Argentine, qui avait permis l'intégration de la population rurale autour des centres urbains en formation à partir des années 1940 (NefFA, 2004), entre en crise avec le premier choc pétrolier. À partir de 1975, l'économie nationale argentine entre dans des cycles d'instabilité et d'inflation qui frappent de plein fouet les classes laborieuses, avec une montée du chômage et de la pauvreté. Les conditions sociales se dégradent sur l'ensemble de la période s'étalant de 1975 à 1990 (avec un pic de dégradation en 1976) : les créations d'emplois déclarés diminuent, le niveau des salaires se détériore et la concentration des revenus s'accentue. Le déclin des salaires réels atteint $37 \%$, à l'échelle nationale, entre 1974 et 1990 (BECCARIA, LOPEZ, 1996). La faible croissance de l'emploi salarié incite les travailleurs à se mettre à leur compte et à ne pas déclarer leur activité. Les faibles revenus caractéristiques des milieux les plus modestes sont particulièrement frappants lorsque l'on compare, pour 1988 , le taux de chômage (de $6 \%$ ) et le taux

(6) Avec d'autres, Jean-Claude BArBIER (2002) propose la notion d'activation pour décrire la variété des politiques publiques de lutte contre le chômage en Europe. de «pauvreté extrême ${ }^{(7)}$ » qui s'élève, quant à lui, à $8,6 \%$ pour la population de la ville de Buenos Aires et à $29,8 \%$ pour celle de sa banlieue. La décennie des années 1980 s'achève dans un contexte d'hyperinflation qui voit la situation sociale se dégrader encore (Collin Delavaud, NefFa, 1994). En 1990, alors que le taux de chômage national est de 8,6\%, la pauvreté touche quasiment la moitié de la population, $42,5 \%$, et la pauvreté extrême $12,5 \%$.

La réduction de moyens alloués aux services publics, soumis aux exigences des politiques d'ajustement portées par le FMI et la Banque mondiale, entre autres, remet en cause ce que Francisco Delich (2002) appelle un «système d'égalité diffuse», qui faisait de l'Argentine un pays égalitaire, où les classes populaires avaient la possibilité de gravir l'échelle sociale, les enfants d'immigrants d'accéder à des études universitaires, et où la classe moyenne connaissait une importance croissante. Ce récit qu'il faut sans doute nuancer (KessLer, 2011) va rapidement devenir obsolète. La part des $20 \%$ de la population qui détiennent les plus hauts revenus ${ }^{(8)}$ passe de $43 \%$ en 1974 à $53 \%$ en 2001 (EQUIS, 2004). Cette tendance rejoint les constats dressés dans les études sur la distribution de revenus dans la région. En Argentine, l'indice de Gini( ${ }^{(9)}$ - indicateur des inégalités de revenus - ne cesse de se dégrader depuis les années 1970 : de 0,33 en 1972 , il atteint 0,43 en $2000,0,47$ en 2002 et 0,50 en 2009 (PNUD, 2012).

Ces tendances vont s'accélérer à partir des années 1990. La loi dite de "convertibilidad», votée en 1991, instaure la parité entre la monnaie argentine et

(7) Selon l'Indec, le taux de pauvreté extrême (indigencia) désigne le revenu minimum en dessous duquel il n'est plus possible de se procurer le «panier alimentaire de base». Le seuil de pauvreté est, quant à lui, atteint lorsque les revenus disponibles ne permettent que de subvenir aux besoins alimentaires; l'accès à d'autres services (école, santé, logement) n'est alors plus garanti.

(8) D'après les estimations d'Alejandro Portes et Kelly Hoffman (2003), s'appuyant sur des statistiques sur les revenus des catégories socioprofessionnelles de la Comisión Económica para América Latina (Cepal; Commission économique pour l'Amérique latine et les Caraïbes), les revenus moyens des travailleurs urbains latino-américains ont diminué ou sont restés stables pendant les années 1990; les revenus moyens des classes dominantes ont eux augmenté plus fortement que dans tous les pays.

(9) «L'indice (ou coefficient) de Gini est un indicateur synthétique d'inégalités de salaires (de revenus, de niveaux de vie...). Il varie entre 0 et 1 . Il est égal à 0 dans une situation d'égalité parfaite où tous les salaires, les revenus, les niveaux de vie... seraient égaux. À l'autre extrême, il est égal à 1 dans une situation la plus inégalitaire possible, celle où tous les salaires (les revenus, les niveaux de vie...) sauf un seraient nuls. Entre 0 et 1 , l'inégalité est d'autant plus forte que l'indice de Gini est élevé.» Source : rubrique «Définitions » de l'Insee; disponible en ligne à l'adresse : http://www.insee.fr/fr/methodes/default. asp?page=definitions/indice-gini.htm; consulté le 17 juin 2014 . 
le dollar américain ${ }^{(10)}$; les privatisations et la dérégulation de l'économie deviennent les maîtres mots de la politique économique du pays. Selon Marcelo Cavarozzi (2012), considérées comme un bien en soi, les privatisations ont été utilisées de manière dogmatique. Les entreprises les plus rentables, comme celles des secteurs des télécommunications ou du transport aérien, n'ont pas été épargnées. Les acteurs internationaux ont apporté un appui croissant au mouvement alors qu'il devenait impossible de faire machine arrière, notamment avec l'arrivée de Domingo Cavallo au ministère de l'Économie en 1991 (Margheritis, 1999). À partir de 1993, le chômage atteint un niveau inédit en Argentine et culmine au taux de $18,6 \%$ de la population active ${ }^{(11)}$ en mai 1995 selon l'Indec. Entre 1992 et 1995, alors que l'emploi total se réduit, le taux d'emploi des couches les moins diplômées de la population (études secondaires incomplètes ou niveau scolaire inférieur) diminue fortement. À partir de 1993, la demande de travail à destination des personnes ayant effectué des études secondaires et universitaires stagne avant de chuter en 1995. Ayant peu de postes à offrir, les employeurs se montrent de plus en plus exigeants et recrutent du personnel de plus en plus qualifié, ce qui, mécaniquement, entraîne une dévalorisation des études les plus modestes. C'est ainsi que les niveaux de chômage les plus élevés sont observés chez les jeunes actifs, avec un pic à 35\% pour les $14-25$ ans en 1995 selon l'Indec. De surcroît, les rémunérations diminuent à partir de 1994, et le travail déclaré stagne (BECCARIA, LoPEz, 1996). L'augmentation du travail non déclaré s'accentue et se poursuit jusqu'en 2003 alors que la croissance commence à repartir ${ }^{(12)}$, pour se stabiliser autour de $40 \%$ en 2010 (NeFFA et al., 2010).

La transformation de l'économie se traduit sur le marché du travail par une augmentation du taux d'activité, notamment des femmes et des jeunes, et en parallèle du chômage et du sous-emploi horaire; les salariés ont un rapport au travail et à l'emploi de plus en plus instable et incertain tandis que le

(10) D'après la loi, toute émission de devises argentines réalisée par la banque nationale doit avoir pour contrepartie une acquisition équivalente de dollars. Le processus de privatisations s'est alors achevé de manière accélérée (HillCoAT, 1994; PANIGo, Torisa ZANe, 2004) afin de garantir l'achat de devises. La nécessité d'atteindre l'équilibre fiscal sera signalée par les autorités comme une justification des réductions des programmes de travail temporaire en dépit d'un chômage croissant. Le principe de convertibilité ne pourra plus être tenu vers la fin de l'année 2001: une des premières mesures du président Duhalde sera d'instaurer une dérogation totale à la loi de convertibilité.

(11) L'Indec produit des statistiques sur l'emploi et le travail, en adaptant les critères du BIT (Bureau international du travail). La population active regroupe population active occupée et population active au chômage.

(12) Selon l'Indec le produit intérieur brut (PIB) a augmenté en moyenne de $7,2 \%$ entre 2003 et 2012 . Information disponible en ligne à l'adresse : http:/www.mecon.gov.ar/basehome/ pdf/indicadores.pdf; consultée le 15 juillet 2014. travail «au noir» et précaire ne cesse de croître. Ceux qui ont un emploi sont contraints d'accepter une dégradation de leurs conditions de travail et d'emploi, telles que le non-paiement des heures supplémentaires ou la réduction du montant de leur rémunération. Les diminutions de salaire, en principe illégales, sont facilitées par les changements d'employeur: les entreprises, notamment petites et moyennes (PME) ${ }^{(13)}$ ayant repris les activités privatisées, ayant une durée de vie de plus en plus courte, les salariés sont amenés à quitter de plus en plus souvent leur emploi. La mobilité des travailleurs d'une entreprise à l'autre s'accompagne de baisses successives de salaire: c'est ce que nous avons constaté lors de notre enquête, dans les secteurs pétrolier, des transports et des télécommunications mais également dans d'autres pans de l'économie - non concernés par les privatisations - comme l'industrie agroalimentaire, l'industrie du papier ou même les services d'entretien. Le secteur public est également touché avec, en 2001, sous le gouvernement de De la Rua (CELS $\left.{ }^{(14)}, 2001\right)$, une réduction de $13 \%$ des salaires des fonctionnaires ${ }^{(15)}$.

Bien que le taux d'activité féminine augmente tout au long des années 1990, celui des femmes mariées reste inférieur de moitié à celui des hommes mariés. En effet, en l'absence d'allocations publiques d'aide à la personne, les dispositifs de garde pour les jeunes enfants, les personnes âgées ou handicapées sont entièrement financés de manière privée. Dans les couches sociales les plus modestes, un grand nombre de femmes prend ainsi en charge ces activités. Aussi, alors que le taux d'activité des femmes mariées dans les couches aisées atteint $64 \%$, il est seulement d'environ 39\% dans les couches populaires (BECCARIA, LOPEZ, 1996).

\section{Transformations du travail et expérience de la pauvreté : la recherche de réponses face à la perte des repères}

Parallèlement à la hausse du chômage, les transformations de l'économie ont affecté les rapports au travail. Les travailleurs font l'expérience d'une dégradation de leurs situations au fil des emplois qu'ils occupent successivement :

«Je travaillais dans une boulangerie à temps plein. Il y avait quatre personnes. Moi j'étais la seule personne déclarée. [...] Maintenant je suis déclaré mais seulement à mi-temps. »

(Gustavo, travaille dans une boulangerie, Berazategui, 2005.)

(13) Selon l'Indec, une petite entreprise emploie jusqu'à cinq salariés et une entreprise moyenne entre six et cinquante.

(14) Centro de Estudios Legales y Sociales, Centre d'études légales et sociales.

(15) Cette réduction des salaires ayant été déclarée inconstitutionnelle, le montant manquant a été remplacé par des titres de dette de l'État argentin (pour les fonctionnaires ayant entamé une procédure judiciaire). 
«Les indemnités, j’ai eu du mal à les avoir. On ne voulait rien me payer. Pour finir, on s'est arrangé avec 3000 pesos. Avec ça, j'ai fait le toit de ma maison, mais pas le sol car c'était trop cher. Dans l'emploi suivant, je n'étais pas déclarée; en fait les derniers mois, personne n'a été payé et nous sommes restés sans travail.»

(Clara, membre du MTD, agent de maintenance [dernier emploi occupé], Berazategui, 2005.)

La perte de travail est liée, dans ces deux cas, à la baisse d'activité de l'entreprise. Pour ceux comme Gustavo qui ont une faible qualification (il n'est pas boulanger), retrouver du travail suppose d'accepter des conditions d'emploi moins bonnes. Ainsi, entre son poste à plein-temps et celui qu'il occupe au moment de l'entretien, il a fait l'expérience d'autres emplois dont il a été contraint de démissionner car les conditions de sécurité y étaient trop mauvaises ou le temps de déplacement depuis son domicile était trop important. Il a ensuite accepté ce travail, à temps partiel, dans une boulangerie proche de chez lui. Célibataire sans enfant à charge, il habite avec ses parents dans la maison familiale qu'il a aidée à construire. Malgré sa diminution, son salaire constitue toujours le revenu principal de sa famille, car ses parents sont allocataires des programmes de travail temporaire (PTT).

Pour Clara, qui n'a suivi que des études primaires, les conditions de travail et d'emploi ont toujours été «compliquées». Elle a ainsi dû se battre pour toucher les indemnités à la suite de la perte de l'emploi déclaré qu'elle occupait. Ensuite, elle a dû se contenter d'un travail au noir mais, au moment de la faillite de l'entreprise dont l'activité de maintenance a été reprise par une autre entreprise, elle n'a pas perçu son dernier salaire. Comme elle l'indique dans l'extrait d'entretien ci-dessus, elle a certes mis à profit la somme reçue en l'investissant dans la construction du toit de sa maison mais elle considère que c'était insuffisant. Sa démarche est caractéristique d'une pratique souvent observée au cours de notre enquête : l'utilisation des indemnités pour améliorer le logement familial. Selon la situation des familles et le montant des indemnités, cette forme d'investissement peut se traduire dans l'achat d'un terrain ou dans celui de matériaux pour construire une partie de la maison (une chambre, les toilettes, le sol ou, comme ici, le toit) ou remplacer des installations considérées comme provisoires.

Les effets des transformations des conditions de travail et d'emploi ne peuvent être pleinement appréhendés que si l'on prend également en compte le degré de stabilité de l'emploi. Le récit de Christian révèle par exemple une précarité accrue qui s'étend même aux emplois déclarés.

Christian: "Quand j'ai terminé là-bas, mon cousin $m$ 'a fait entrer dans la peinture, c'est-à-dire qu'on est devenu peintre, chez Pétrochimique; j'ai commencé là-bas et j'y ai travaillé cinq ans. »
Pìa Rius : «Comment était ton travail chez Pétrochimique?»

Christian: "De 8 heures à 16 heures, du lundi au vendredi. »

Pìa Rius : "Est-ce que tu connaissais le travail ou tu l'as appris là-bas?»

Christian: "Non, je l'ai appris là-bas, mon cousin me l'a appris. J'ai travaillé un an dans cette entreprise et après un nouvel appel d'offres, une autre entreprise l'a emporté et je suis resté travailler chez eux pendant trois ou quatre ans. »

(Christian, membre du MTD, dernier travail comme peintre, Berisso, 2004.)

Christian, relativement habitué au travail non déclaré, a tendance en début d'entretien à minimiser son expérience du chômage (" $j$ 'ai travaillé cinq ans »); puis il se reprend pour détailler les périodes de chômage qui séparent les deux activités dont il est ici question(16). Les frontières entre travail et non-travail semblent poreuses : elles affectent non seulement les conditions d'emploi (par la succession des contrats de courte durée, l'augmentation de l'arbitraire pour certains travailleurs qui perdent leur poste et pas d'autres), mais aussi la perception de ce qu'est le travail et son contraire, l'absence de travail (Rius, 2007). Une telle instabilité concerne également la rémunération, dont le montant fluctue au cours du temps comme on peut le voir dans la suite de l'entretien :

Pìa Rius : "Tu te rappelles combien tu gagnais?»

Christian: "Je gagnais 450 à 500 pesos selon les mois. C'était la moitié déclarée et la moitié au noir.»

Pìa Rius : "Et tu étais d'accord avec ça?»

Christian : «Oui, parce qu'on ne travaillait pas trop. Il nous fallait parfois peindre tant de mètres par jour, c'est tout. J'ai même touché 600 à 700 pesos par mois grâce aux heures supplémentaires. En fait, on nous payait par quinzaine, 250 à 300 pesos. »

Pìa Rius: "Tu pouvais toujours faire des heures supplémentaires?»

Christian: "Quand il y en avait, si je voulais, je pouvais les faire; mais il n'y en avait pas toujours. "

Ce que l'on peut considérer comme des anomalies de l'emploi semblent intégrées au quotidien des travailleurs : Christian ne trouve apparemment pas problématique ni le fait de percevoir la moitié de son salaire «au noir» ni que son niveau de revenu ait ainsi été soumis à la possibilité de faire des heures supplémentaires selon les besoins de l'entreprise. Après un premier contrat à Pétrochimique et une période de chômage, Christian a été repris par l'entreprise ayant repris l'activité de la première (elle a remporté le nouvel appel d'offres). Comme dans les cas de Gustavo et de Clara, la perte de travail n'est

(16) Pour l'étude de la temporalité dans l'expérience de chômage, voir notamment LAZARSFELD et al., 1981 ; SCHNAPPER, 1981; LE MouËL, 1981. 
pas la conséquence d'une activité qui s'arrête mais d'une insertion instable dans l'emploi.

Notre enquête montre que, l'ensemble des personnes rencontrées, qu'elles soient ou non en emploi, s'attend à ce que des études secondaires facilitent l'accès au marché du travail. Les jeunes ayant fini leurs études ne comprennent pas pourquoi ils ne trouvent pas de travail ou sont déçus des postes proposés (maintenance, services à la personne). Les exigences des employeurs sont vécues sur le mode de l'absurde par les travailleurs surdiplômés, qui ne comprennent pas qu'il leur soit demandé d'avoir suivi des études secondaires pour obtenir un poste manuel et non qualifié, de laveur de voitures par exemple. Exiger désormais des candidats qu'ils soient diplômés pour occuper des postes peu qualifiés a pour conséquence de désavantager particulièrement les jeunes des milieux populaires qui, souvent, quittent l'école très tôt pour travailler au noir. C'est la situation d'un des membres du MTD de Berisso, Alejo :

«Je suis toujours en train de chercher du travail, je parle aux gens que je connais, je lis aussi les journaux, les petites annonces, pour voir s'il y a du travail et il y a du travail, mais tu dois avoir suivi l'école secondaire et tout cela, et je ne l'ai pas fait. C'est-àdire que pour moi c'est encore plus compliqué, même pour laver des voitures on te demande l'école secondaire. Et si tu ne l'as pas faite, la situation devient un peu compliquée.»

(Alejo, membre du MTD, remplaçant à l'accueil dans un taxiphone, Berisso, 2004.)

L'entretien ci-dessus montre que les personnes sans-emploi multiplient les canaux de recherche : participant au collectif des desocupados, Alejo ne manque pas de recourir à des stratégies plus classiques de recherche d'emploi et «d'auto-emploi». Depuis qu'il a commencé à travailler, il est dans un état d'alerte permanent, constamment à la recherche de postes qui constituent autant de sources de revenus supplémentaires. D'origine rurale, il travaille depuis son plus jeune âge, toujours au noir, ce qui l'a empêché de suivre des études. S'il s'est inscrit à l'école pour adultes afin de pallier ce manque, il reste sceptique quant à la possibilité réelle de pouvoir y consacrer du temps. Outre l'activité liée au programme de travail temporaire, il fait des remplacements dans un taxiphone - qu'il utilise par ailleurs pour rechercher du travail via internet et fournit les commerces des environs en produits de nettoyage qu'il achète en gros dans la ville voisine de La Plata.

\section{L'émergence des acteurs collectifs face à la crise du travail}

Au début des années 1990, la question de l'emploi est au centre des préoccupations des Argentins. Les enquêtes d'opinion élaborées par le sociologue Francisco Delich (2002) soulignent alors que 22\% des sondés la considèrent comme leur problème le plus important; en 1999, le chômage est devenu la préoccupation principale de la moitié des personnes consultées (Delich, 2002). Du côté des politiques publiques, l'allégement des charges sociales et l'instauration d'une période d'essai sont présentés comme des outils censés faciliter la création d'emplois. Pour autant, les programmes sociaux à destination des populations au chômage restent marginaux et sans ampleur au regard des difficultés rencontrées.

Les réactions face à l'évolution de la politique du gouvernement argentin ne sont pas homogènes. L'étude de James McGuire (1996) sur les grèves nationales montre que la forte mobilisation des travailleurs n'a pu empêcher la décision de privatisation de l'entreprise nationale de téléphonie (première touchée par les réformes). Des divisions voient le jour entre syndicalistes soutenant et s'opposant au gouvernement «menemista », l'opposition étant conduite par le Mouvement des travailleurs argentins (MTA) et la Centrale des travailleurs argentins $(\mathrm{CTA})^{(17)}$. Par ailleurs, l'interdiction des grèves dans le secteur public est promulguée dès le mois d'octobre 1990. La loi sur l'emploi (Ley de empleo $\mathrm{n}^{\circ} 2184 / 90$ ) entérine la création de nouveaux types de contrats temporaires et d'un système d'assurance chômage (seguro de desempleo), qui ne couvre toutefois bien sûr pas les pans entiers de la population argentine qui travaillent dans le secteur informel. La stabilité de la monnaie et sa parité avec le dollar étant devenues prioritaires, la définition du rôle de l'État, y compris de l'État-Providence, semble y être subordonnée. L'ensemble de ces facteurs a des répercussions sur la place occupée par l'emploi dans l'accès aux droits sociaux.

Si les mobilisations pour empêcher les privatisations échouent, celles qui sont ensuite organisées dans des localités pétrolières en 1996 et 1997 pour réclamer de nouvelles politiques de lutte contre le chômage prennent une ampleur nationale et obligent le gouvernement à intervenir pour désamorcer le conflit. Ces actions, qui consistent à dresser des barrages et bloquer les routes, valent aux participants d'être baptisés piqueteros par les journalistes, dénomination qu'ils adoptent ou refusent tour à tour. Maristella Svampa et Sebastiàn Pereyra (2004)

(17) En octobre 1989, alors que Carlos Menem est à la tête du pays depuis quelques mois, la division est officialisée par la création de deux entités séparées, la CGT Azopardo et la CGT San Martin, proche du gouvernement. Fin 1992, la CGT se réunifie mais plusieurs courants subsistent et s'y affrontent : d'un côté, une grande majorité des leaders syndicaux participe activement à la mise en œuvre des réformes gouvernementales ; d'un autre, ceux qui représentent des voix syndicales en désaccord avec le gouvernement mais n'ayant pas coupé les liens avec le parti péroniste, créent le Mouvement des travailleurs argentins (MTA); enfin, ceux qui s'opposent au modèle social et économique mis en œuvre par le gouvernement fondent le Congrès des travailleurs argentins (CTA) (PALOMINO, 1995, pp. 203-230). 
définissent trois types de «mouvement de piqueteros», avec des lignes revendicatives distinctes : une ligne politique, une ligne syndicale et une ligne territoriale.

SVAmpa et Pereyra situent dans la catégorie «ligne politique» les organisations dont les modalités d'action sont empruntées aux partis politiques de gauche. C'est par exemple le cas du Polo Obrero (Pôle ouvrier) ou du Movimiento Teresa Vive (Mouvement Thérèse Vivante, qui adopte le nom d'une enseignante tuée lors des manifestations à Neuquén), tous deux d'inspiration trotskiste, ou du Movimiento Tierra y Liberación (Mouvement Terre et libération), lié au parti communiste argentin. Notons que les positions de ces collectifs au sujet des programmes de travail temporaire (PTT) ${ }^{(18)}$ fluctuent au cours du temps: le Polo Obrero commence ainsi par refuser les programmes de travail temporaire (PTT) qui, d'après lui, sont en réalité des contrats de travail précaire, sous-payé et sans cotisations à la sécurité sociale avant d'accepter les allocations versées dans le cadre de ces programmes comme un palliatif(19).

D'après Svampa et Pereyra, ce qui caractérise les mouvements sociaux relevant de la «ligne syndicale» c'est leur composition interne, comme celle de la Corriente Clasista Combativa (Courant de classe combatif) et de la Federacion Tierra y Vivienda (Fédération pour la terre et l'habitat), liées à la centrale syndicale dissidente, CTA (Congrès des travailleurs argentins) (20). Qu'il s'agisse de leurs porte-parole, désignés par le terme de «référents», ou de leur base, ces mouvements sont constitués d'anciens travailleurs industriels, d'anciens travailleurs précaires, de femmes qui ont peu participé au marché formel du travail et de jeunes sans expérience de travail (formel ou informel). La distinction avec les organisations développant une ligne territoriale n'est pas tant la population qui les compose, que les liens existant avec la centrale syndicale. En effet, comme celles de la ligne territoriale ces organisations se nourrissent d'autres expériences telles que celles des «communautés ecclésiastiques de

(18) Lorsqu'ils acceptent d'y participer, les desocupados adhérant à ces programmes doivent accomplir, en contrepartie du versement de l'allocation, une activité dite «contrepartie de travail» au profit d'une collectivité publique, d'un établissement public ou d'une association, d'une durée comprise entre quatre et six heures par jour.

(19) Seul un mouvement de desocupados (MTD) à La Matanza, regroupant une cinquantaine de membres, persiste dans son refus des allocations au fil du temps; il reçoit en revanche des donations d'entreprises et d'organisations non gouvernementales $(\mathrm{ONG})$.

(20) Cf. supra note 17. base $^{(21)} 》($ SAnTILLAN, 2008) ou des « occupations de terrain» dénommées asentamientos (22) (MerkLeN, 2005).

Enfin, la «ligne territoriale» regroupe les organisations qui ne relèvent de manière organique d'aucune des deux lignes précédentes et s'inscrivent plutôt dans des traditions locales comme, par exemple, les MTD de la banlieue de Buenos Aires ou l'Union des travailleurs Desocupados, dans le nord du pays, à Salta.

Quelle que soit leur origine, la plupart de ces organisations comptent dans leurs rangs des femmes et des jeunes qui ont peu ou pas travaillé dans le secteur formel. La recherche ethnographique permet de constater que ces réseaux s'appuient sur des appartenances locales et familiales. Au sein d'une même famille (Quirós, 2006) et au cours du temps (RIUS, 2010), différents collectifs sont mobilisés par des membres passant d'un réseau à un autre en fonction des logiques locales. Les soutiens familiaux sont d'ailleurs bien visibles lors des épisodes de mobilisation : les femmes sont dans la rue avec leurs enfants en poussette; des repas collectifs sont partagés pendant les manifestations; chaque groupe a confectionné son propre drapeau qui indique à la fois son appartenance à une organisation et son appartenance locale (par exemple MTD de Berisso, MTD de Berazategui, etc.). Les participants vivent ces mobilisations de manière ambivalente : d'un côté, les femmes signalent qu'elles constituent pour elles la possibilité de « sortir du quartier », de « sortir

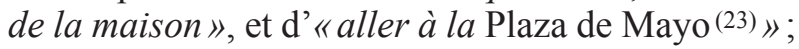
d'un autre, elles mettent en avant les efforts et sacrifices auxquels elles consentent pour "faire face au froid et à la chaleur», "marcher pendant de longs kilomètres tout le long de la journée», "surmonter [leur] peur», notamment quand il y a un risque de répression policière. Les pneus brûlés caractéristiques des premiers piquetes à l'époque où les manifestations ont lieu aux portes ou à l'intérieur de la ville de Buenos Aires, sont devenus le symbole de la lutte. Les projets portés par chaque groupe sont différents les uns des autres mais dirigeants et délégués soulignent avec fierté que tous ont été l'oc-

(21) Laura Santillan (2008) explique que la présence des communautés ecclésiastiques de base dans les quartiers populaires de la banlieue de Buenos Aires dans les années 1980 est liée à un engagement religieux hors structure paroissiale, qui consiste notamment en des lectures collectives de la Bible et des analyses de la réalité des populations.

(22) Denis Merklen (2005) précise que les asentamientos sont certes des occupations illégales de terrains mais que, contrairement aux bidonvilles (villas), elles cherchent d'emblée à acquérir la légitimité de véritables quartiers urbains (barrios) grâce à un travail de coordination accompli par des délégués qui contribuent ainsi au processus de leur insertion sur le territoire ou «inscription territoriale».

(23) Situé en face de la Casa Rosada, où siège le pouvoir national, cette place historique représente le demos dans l'espace public. Silvia Sigal (2006) en propose une chronique des différents usages. 
casion de développer de nouvelles compétences : les «compañeros savent pourquoi ils sont là» et manifestent de manière organisée.

\section{Traitement public du chômage et de la mobilisation des desocupados}

Lors des débats sur le chômage dans l'Argentine des années 1990, le marché apparaît comme le seul mécanisme susceptible de résoudre ce problème (KesSLER, 1996). Les dommages sociaux causés par le chômage sont, quant à eux, assimilés à ceux d'une épidémie; l'utilisation d'une telle métaphore de type médical tend à nier l'impact des réformes économiques en cours sur le chômage. La période est par ailleurs à la culpabilisation des individus (GonzÁlez Bombal, Leoni 2003), puisqu'en raison de leurs caractéristiques personnelles, ils sont considérés comme responsables de leur situation. C'est ainsi le manque de "flexibilité» (ne pas accepter «n'importe quel travail») et de faculté «d'adaptation» qui est identifié comme cause du chômage.

\section{Les programmes de travail temporaire (PTT)}

Les premiers programmes de travail temporaire (PTT) - conçus par le gouvernement Menem consistent en des actions de courte durée, qui proposent de trois à six mois d'indemnité, dont le montant est inférieur à celui des salaires précédemment perçus de sorte à éviter toute incidence sur le niveau des rémunérations dans le pays et à ne pas provoquer d'effet désincitatif à la recherche d'emploi. Il ne s'agit plus d'investir dans l'économie à partir des entreprises d'État mais de promouvoir la construction d'infrastructures locales dans des zones désignées comme prioritaires, avec un financement municipal(24). Les organisations de desocupados proposent leurs propres projets (cantines populaires, boulangeries, jardins potagers, ateliers de couture ou de menuiserie), dont les activités sont en partie accomplies par leurs membres, y compris s'agissant des tâches administratives exigées par l'État pour le versement des allocations (dossiers d'inscription, fiches de présence, etc.) ${ }^{(25)}$.

Le premier programme Trabajar est alors considéré par le ministère du Travail et de la Sécurité sociale comme un «programme d'emploi direct». Il a pour objectif de verser un revenu aux travailleurs pauvres pendant leurs périodes de recherche d'emploi et de contribuer au développement des

(24) Les municipalités n'ayant pas toujours disposé des ressources nécessaires pour faire face à l'ampleur du chômage, les travaux d'infrastructure se résument parfois à des activités de défrichage.

(25) Ces personnes sont considérées comme occupées. Cf. supra, note 11 . structures économiques et sociales des communautés par le biais de projets locaux. Le programme est mis en œuvre sur l'ensemble du territoire national, et devient une monnaie d'échange dans les rapports entre les autorités et les organisations des travailleurs desocupados, qui ont précisément créé des collectifs afin d'accéder à ces "emplois transitoires ». La rétribution des travailleurs ne constitue pas un salaire et exempte tant les «employeurs» que les «travailleurs» des charges sociales usuellement requises par la législation (sécurité sociale, cotisation-retraite, etc.) ${ }^{(26)}$. Pendant une période de six mois, les desocupados «participant» au programme (autrement appelés «bénéficiaires» ou « ayant droits » (27)) perçoivent une somme comprise entre 160 et 200 pesos.

Le programme est destiné à ceux qui n'ont pas ou peu de qualifications et rencontrent davantage de difficultés dans l'accès à l'emploi. Les desocupados qui y sont admis, sélectionnés sans distinction de sexe, doivent être âgés de plus de 16 ans et ne pas percevoir de prestation chômage ou toute autre allocation publique. Chaque projet inclut entre 5 et 40 travailleurs. Un critère requis supplémentaire est fondé sur la condition familiale : $50 \%$ des titulaires doivent être chefs de famille. Conçus dans le cadre des prêts de la Banque mondiale, les programmes Trabajar font l'objet d'un réexamen tous les ans, après avoir été évalués par SIEMPRO (Sistema de evaluación $y$ monitoreo de programas sociales), le bureau d'évaluation des programmes sociaux créé dans le cadre du ministère du Développement social sur la base d'un financement par les organismes internationaux. Ses évaluations (1997-1998) estiment que le programme a atteint l'objectif sur lequel il s'était focalisé : $40 \%$ des allocataires appartenaient aux foyers des $5 \%$ les plus pauvres de la population; d'autres évaluations vont dans le même sens (BRUSCO et al., 2005).

La décision d'approbation des projets appartient au ministère du Travail, mais la présentation des projets est ouverte aux ONG locales -y compris aux organisations de desocupados constituées en associations - et intègre un plan de formation. Les premières années, $85 \%$ des titulaires recensés étaient des hommes et $15 \%$ des femmes. En 1998, le rapport d'évaluation rend explicite la volonté du gouvernement d'inclure une proportion plus élevée de femmes. L'inclusion

(26) Le programme prévoyait l'inclusion d'un système de sécurité sociale pendant la réalisation des projets mais cette dimension n'a pas été mise en œuvre.

(27) Nous ne retenons toutefois pas ces termes. En effet, d'une part le terme «bénéficiaire», plus proche de celui utilisé dans les documents des ministères (sujeto beneficiario), souligne la mise sous tutelle d'un individu réduit à la dépendance vis-à-vis de l'État. D'autre part, les conditions d'accès aux programmes de travail temporaire (PTT) et de maintien dans ces dispositifs sont marquées de pratiques arbitraires si bien que le terme d'«ayant droit» ne peut être plus qu'un euphémisme. 
des cantines populaires comme projets agréés répond à cet objectif (BANQUE MONDIALE, 1997).

Si les programmes de travail temporaire (PTT) sont considérés, notamment par la presse, comme des créations de «postes de travail»(28) et une relance des chantiers de travaux publics, cette modalité d'intervention caractéristique d'un Étatprovidence par ailleurs fortement questionnée depuis le lancement du processus de privatisation ne correspond pas aux projets mis en place par les programmes effectifs. À travers une première génération de programmes de travail temporaire (PTT) ${ }^{(29)}$, le gouvernement accorde les allocations aux chômeurs dans le cadre de projets d'infrastructures locales pour lesquels les mairies doivent financer les matériaux et outils de travail, ce qui en réduit de fait la portée.

\section{L'évolution des PTT et de leur application au gré des changements politiques}

Suite à la démission du Président De la Rua fin $2001^{(30)}$ et à la nomination d'Adolfo Rodriguez Saá (31) à la tête du gouvernement, ce dernier propose la création d'un programme qui vise la création d'un million de «postes de travail» à partir de la reconnaissance d'un «droit au travail digne». Rodriguez Saá est contraint à la démission mais le programme est mis en place par son successeur.

Eduardo Duhalde, dans son discours d'investiture présidentielle donne la priorité à la «paix sociale». Début janvier 2002, lorsque le peso est dévalué, s'achève la période dite du modèle de convertibilité. Dans le cadre d'une «conférence sociale argentine» (mesa de diálogo argentino), le gouvernement convoque des syndicats, des entrepreneurs de l'Union industrielle argentine (UIA), la Sociedad Rural, des fonctionnaires de l'ONU et des représentants des différentes religions ${ }^{(32)}$. Si les

(28) Cf. par exemple Auyero (2002), KlachKo (2002), Svampa, PEREYRA (2004).

(29) Les différentes générations de programmes de travail temporaire peuvent fonctionner de manière juxtaposée sans se remplacer complètement.

(30) Le départ du président crée une situation de vacance du pouvoir. Rodriguez Saá est nommé président intérimaire en charge d'organiser le processus électoral mais il échoue à obtenir le consensus du parti péroniste dont il est issu. Il est amené à démissionner et Eduardo Duhalde, ancien gouverneur de la province de Buenos Aires et alors sénateur du même parti, est désigné par le Sénat pour achever le mandat entamé par De la Rua.

(31) Discours devant l'Assemblée législative, 23/12/2001, cité par Di Leo (2003).

(32) Les institutions participant à cette conférence sont centrales dans l'économie et la société argentine. L'Église a participé à des espaces de conciliation lors des premiers piquetes; l'industrie nationale a été privilégiée par la dévaluation de la monnaie et est un des moteurs de l'activité à partir de 2003, ainsi que l'activité agricole et l'élevage, historiquement présents. Le caractère latifundiste de la structure agraire argentine s'est renforcé au cours des années 1990 avec l'introduction, notamment, du soja transgénique (Goulet, Hernandez, 2011). organisations de desocupados n'en font pas partie, certaines sont reçues par le président Duhalde ou par des ministres et d'autres encore participent à des discussions qui s'inscrivent, elles aussi, dans cet espace de dialogue. Au cours du mois de février 2002, l'état d'urgence est déclaré dans les domaines de la santé, la sécurité alimentaire et de l'emploi (33). Un budget spécial est alloué afin de distribuer nourriture et médicaments. Malgré la dévaluation, le montant des allocations reste inchangé; le nombre des programmes de travail temporaire de seconde génération, les programmes "Chefs et cheffes de ménage au chômage»-Plan Jefes y Jefas de Hogar Desocupados (PJJHD) - est multiplié par douze puis par deux pour dépasser les deux millions de desocupados-allocataires en 2003 (cf. tableau 1).

Tableau 1 : Programmes de travail temporaire et capacitation (PTT)

\begin{tabular}{|c|c|c|c|}
\hline Gouvernement & Période & PTT & $\begin{array}{c}\text { Nombre moyen de } \\
\text { titulaires }\end{array}$ \\
\hline \multirow{3}{*}{$\begin{array}{l}\text { Carlos Menem } \\
\text { (PJ) }\end{array}$} & 1996 & Trabajar I & 82000 \\
\hline & 1997 & Trabajar II & 130000 \\
\hline & 1998 & Trabajar III & 118000 \\
\hline \multirow{3}{*}{$\begin{array}{l}\text { Fernando } \\
\text { De la Rua } \\
\text { (Alianza) }\end{array}$} & 1999 & \multirow{3}{*}{ Trabajar III } & 106000 \\
\hline & 2000 & & 86000 \\
\hline & 2001 & & 91800 \\
\hline \multirow{2}{*}{\begin{tabular}{|l|} 
Eduardo \\
Duhalde (PJ)
\end{tabular}} & 2002 & \multirow{2}{*}{ JJHD } & 1126000 \\
\hline & 2003 & & 2128400 \\
\hline \multirow{4}{*}{$\begin{array}{l}\text { Nestor } \\
\text { Kirchner (PJ) }\end{array}$} & 2004 (1) & \multirow{4}{*}{\begin{tabular}{|l|} 
JJHD (2) / \\
Familles \\
pour l'inclu- \\
sion sociale*
\end{tabular}} & 1919900 \\
\hline & 2005 & & $1500000 / 237014^{* *}$ \\
\hline & 2006 & & $1000000 / 323138$ \\
\hline & 2007 & & $800000 / 454372$ \\
\hline
\end{tabular}

1. Pour l'année 2004, sont considérés les programmes suivants : Jefes de Hogar, PEC et Recuperación productiva.

2. À partir de 2003, les titulaires du JJHD sont orientés vers des nouveaux programmes (INDEC, 2007).

* Les données de ce programme ont été publiées par le CELs (2005) suite à une demande de renseignements émanant de cette ONG invoquant le droit à l'information. Décimo Informe (2005) du Conseil de consultation nationale présente les mêmes données.

** Le premier chiffre correspond au nombre moyen des titulaires du JJHD et le second à celui du programme Familles.

Lecture : En 1996, sous le gouvernement péroniste de Carlos Menem, le programme Trabajar I distribuait une moyenne de 82000 allocations. Source : Élaboration à partir des données du MTEySS (Ministerio de Trabajo, Empleo y Seguridad Social), Secretaría de Empleo, Area de monitoreo.

Cette deuxième génération de programmes donne à la protection sociale une dimension familialiste autour de la figure du "chef de ménage». D'une part, l'expérience de la première génération des programmes Trabajar a structuré le tissu associatif et institutionnel permettant de couvrir l'ensemble du territoire national. D'autre part, les

(33) Decreto de necesidad y urgencia (DNU) 108/02, DNU 486/02 et DNU 165/02 respectivement. La déclaration de l'état d'urgence confère provisoirement au président des facultés relevant du pouvoir législatif, des compétences sur le budget par exemple. 
débats préalables ainsi que les propositions développées dans différents espaces de la société civile, comme par exemple le projet développé par le Front national contre la pauvreté, Frenapo ${ }^{(34)}$, montrent la légitimité de l'intervention sous la forme d'un «droit social ${ }^{(35)}$ ». Laura Golbert $(2003$, p. 18) souligne néanmoins un problème d'iniquité concernant l'accès effectif à ce programme : à partir de l'enquête statistique sur les ménages (EPH-Indec) du deuxième trimestre de 2003, cette sociologue constate que seul un foyer sur trois en dessous du seuil de «pauvreté extrême» a accédé au programme; pour les foyers en dessous du seuil de pauvreté, la proportion n'est plus que d'un sur douze, sachant que les allocations versées sont loin de suffire pour sortir des situations de pauvreté(36). D'après les autorités de Caritas qui siègent dans la commission de contrôle du programme ${ }^{(37)}$, environ 400000 familles en situation de pauvreté extrême - normalement en condition de recevabilité pour ces programmes - ont été écartées par sa «fermeture» en $2002^{(38)}$. La ministre du Travail explique alors qu' «à un moment donné, il fallait établir une ligne de restriction pour des raisons de budget ${ }^{(39)}$ ». L'organe consultatif n'a aucun caractère décisionnel, néanmoins, dans ses recommandations de mars 2003, il soutient que «la suspension des inscriptions a entraîné une grave entorse aux dispositions du décret 565/2 [décret de création du programme]».

Une différence importante introduite par ce programme Chefs et cheffes de ménage, comparé au précédent programme Trabajar, est l'introduction du critère de «responsabilités familiales». En raison des critères d'éligibilité au programme, entre autres l'obligation d'avoir une personne à charge et d'être soutien de famille, les personnes

(34) Frente nacional contra la pobreza soit Front national contre la pauvreté. Il s'agit d'un courant non partisan auquel participent des députés, des dirigeants syndicaux et des intellectuels.

(35) Confronté aux accusations de clientélisme concernant les programmes de travail temporaire (PTT), le ministre du Travail a désigné les conseils consultatifs (consejos consultivos locales) comme espace institutionnel destiné à garantir la transparence.

(36) Le programme prévoit 150 pesos par famille alors que les besoins d'une famille type (selon l'Indec, deux adultes et deux enfants) sont de 332,36 pesos pour ne pas être considéré sous le seuil de pauvreté extrême et de 727,88 pesos pour ne pas tomber sous le seuil de pauvreté (le Smic est alors de 300 pesos et le salaire moyen de 400 pesos; CTA, 2003).

(37) La CONAyEC est une commission de contrôle créée de manière ad hoc pour cette occasion.

(38) Ce même rapport consigne que, depuis mai 2002, la Defensoria del pueblo a reçu pas moins de 600 plaintes individuelles et 2600 plaintes collectives pour motif de refus d'inscription. Source : La Nación, 19 octobre 2004, « Impiden a desocupados que se inscriban en el plan jefes y jefas ».

(39) Déclarations en conférence de presse par la ministre du Travail du gouvernement Duhalde, Graciela Camaño, au sujet de la fermeture officielle des registres du PJJHD. Source: Página/12, "Un universal muy particular. Limitan inscripciones en el Plan Jefes de Hogar», 12 juin 2002, p. 7. de plus de 18 ans sans enfants et les personnes âgées sont a priori exclues du programme. Les jeunes célibataires qui vivent chez leurs parents ne peuvent notamment plus prétendre avoir accès à des allocations. Une autre condition imposée par ce programme pour ces «chefs de ménages pauvres» est que le conjoint ne perçoive ni salaire ni revenu issu des aides sociales ${ }^{(40)}$. Cette condition permet de radier un titulaire du programme dès lors que lui ou son/sa conjoint/e a un emploi déclaré. Cela se reflète dans la composition des foyers concernés par le programme, dont une grande partie est constituée de foyers monoparentaux ${ }^{(41)}$. Par ailleurs, suite à la dévaluation de la monnaie en 2002, le montant de l'allocation n'a pas été actualisé. Des ONG comme le Centre d'études légales et sociales (CELS) ont présenté des réclamations par la voie judiciaire pour restituer leur droit à l'allocation à certains ayants droit et ont obtenu gain de cause. Le gouvernement a alors prolongé sur l'année le programme, mais le nombre d'allocataires a quand même continué de diminuer.

Notre recherche auprès des organisations de desocupados souligne le contournement des contrôles exercés par l'État national et provincial dès les premières mobilisations. Dans le même temps pourtant, les mouvements de desocupados (MTD) se sont mobilisés pour obtenir la reconnaissance des droits des travailleurs. Ainsi, fin 2003, suite à une campagne de mobilisation du réseau des organisations, le gouvernement national a octroyé un treizième mois d'allocation pour le mois de décembre, opération qui sera renouvelée, un an plus tard avec un montant réduit de moitié, soit 75 pesos ${ }^{(42)}$, puis abandonnée en 2005.

Par ces programmes, le gouvernement a alloué des ressources à une partie des populations en difficulté, mais sans conférer pour autant un statut juridique aux travailleurs concernés. La politique menée traite notamment de façon différente les titulaires selon leur situation individuelle (âge, situation familiale, niveau d'étude). Ce trait va se renforcer dans les programmes de travail temporaire de troisième génération puisque les titulaires des allocations sont invités à s'inscrire dans des nouveaux programmes dont le montant de l'allocation est augmenté (jusqu'à 250 pesos), soit sous l'égide du ministère du Travail et de la Sécurité sociale à travers le programme Mas y mejor trabajo (des emplois meilleurs et en plus grand nombre),

(40) Les allocations familiales en Argentine ne sont alors distribuées qu'aux seuls salariés.

(41) On dénombre ainsi $20 \%$ de titulaires célibataires et $27 \%$ qui sont veufs ou divorcés. Source : Seconde enquête d'évaluation du PJJHD menée en juin 2004 dans la ville de Buenos Aires, Santa Fe, Tucumán, Córdoba, Formosa et prov. de Buenos Aires, MTEySS, Subsecretaría de Programación Técnica y Estudios Laborales, Dir. Gral. Estadísticas y E. Laborales (MTEySS, 2004).

(42) DNU1668/04. CELS, 2005, 330. 
soit dans l'orbite du Développement social. Avant de nous attarder sur ces changements successifs, nous précisons les processus d'appropriation des programmes de travail temporaire des deux premières générations.

\section{Les mouvements des desocupados et la conception des programmes de travail temporaire : reconnaissance limitée et marges de manœuvre étendues}

S'il existe, le lien entre l'augmentation des blocages de route par les desocupados et le volume des allocations distribuées dans le cadre des PTT ne peut être réellement considéré comme un facteur déterminant des politiques publiques de l'emploi en Argentine. Les blocages constituent cependant un élément important de contexte pour comprendre les conditions politiques d'élaboration et le fonctionnement du premier programme Trabajar ainsi que des programmes successifs. Du point de vue des secteurs mobilisés, la sélection des projets par le programme exclut de fait ceux qui ne sont pas entrés en contact avec les réseaux de desocupados. En ce sens, l'évolution des programmes de travail temporaire constituerait autant une réponse à la mobilisation sociale ${ }^{(43)}$ qu'aux situations de pauvreté.

«[Je suis au MTD depuis 1997] parce que je n'avais pas de travail. La lutte des gens du quartier commençait. Nous luttions pour l'unité entre les voisins et le vrai travail, autrement nous ne trouvions rien. Cela faisait quasiment deux ans que je n'avais pas de travail. La seule solution que je voyais, c'était la lutte. [...] Nous étions dans une cantine populaire, j'y suis restée pendant six mois jusqu'au moment où j'ai eu le poste de travail.»

(Beto, MTD La Veron, février 2003.)

«J'ai commencé en 1999, par une compañera. Ayant mon fils en bas âge elle m'a dit: "Va là-bas, ils vont t'inscrire et tu vas obtenir un plan. " [...] À ce momentlà, mon partenaire avait un travail, tout. Peut-être que c'est pour ça qu'il ne voulait pas que j'y aille. [...] Deux mois plus tard, mon partenaire a été viré. Nous nous sommes attachés au mouvement. C'était très important, je suis restée un an comme desocupada car il n'y avait pas de poste. 》

(Julia, déléguée MTD La Veron, décembre 2003.)

Ces entretiens évoquent les difficultés d'accéder aux allocations du programme Trabajar. Ce qui fait de la mobilisation, «la lutte», la seule voie d'action possible. Les évaluations réalisées durant cette période (SIEMPRO, 1998) montrent une distribution des allocations en accord avec la formulation du Trabajar. Pourtant, cette déléguée souligne qu'elle est demeurée desocupada

(43) $C f$. la déclaration du Président par intérim Duhalde qui indique que la création du programme cherche la «paix sociale» (DI LEO, 2003); plusieurs études vont dans ce sens (Svmpa, Pereyra, 2004; Golbert, 2006). durant un an : elle faisait partie du groupe comme bénévole ou militante, mais elle ne recevait pas d'allocation de ce programme de première génération; elle n'en aura finalement une que par l'intermédiaire du MTD. Ce dont témoignent nombre d'entretiens, c'est l'injustice, pour ceux qui en sont exclus, des critères d'éligibilité institués par le gouvernement.

Les organisations de desocupados ont mobilisé leurs membres afin que soient intégrées leurs attentes lors de l'élaboration des programmes de travail temporaire, notamment à l'occasion d'une marche massive de La Matanza à Plaza de Mayo en $2002^{(44)}$. Un des leaders, Luis D'Elia, avance plusieurs revendications, dont l'accès des jeunes et des personnes âgées à l'allocation dont ils sont exclus par les critères familialistes du programme Chefs et cheffes de ménage. Lors d'une réunion avec le président Duhalde, Luis D'Elia soulève aussi la récupération par les partis politiques de ces programmes, à travers la sélection des bénéficiaires. Les programmes d'emplois communautaires, Programa de Empleo Comunitario (PEC), sont alors créés de manière complémentaire au programme Chefs et cheffes de ménage : ils sont négociés sous forme de quotas entre secteurs bénéficiaires qui correspondent aux principaux groupes mobilisés, et le gouvernement national afin d'assurer l'accès des membres des organisations aux dits programmes ${ }^{(45)}$. Le président Duhalde évoque alors dans son discours officiel un «droit social» et non pas seulement un palliatif à l'urgence de la situation (46). Il déclare "l'universalisation» des programmes de travail temporaire afin «d'assurer un revenu minimal mensuel à toutes les familles argentines ».

$\mathrm{Au}$ niveau local, les organisations présentent leurs propres projets et décident des activités mises en œuvre dans le cadre du programme; l'organisation et la division du travail lié à ces activités (recherche des budgets, achat des matières premières, relations avec d'autres organisations, temps de présence aux activités, présentation des documents aux autorités) font l'objet de débats internes aux groupes. Par exemple, des personnes issues d'une même famille peuvent accéder à des programmes de travail temporaire (PTT) sur des fonctions spécifiques telles que la lutte («la lucha»), le temps bénévole consacré aux organisations et aux mobilisations collectives. Ceux qui sont exclus par l'évolution du programme sur des critères familialistes sont réintégrés de fait par

(44) Pagina 12, février 2002.

(45) D'après Laura Golbert (2006), les négociations concernent l'ensemble des programmes de travail temporaire (PTT).

(46) Le président Duhalde annonce publiquement le programme en avril 2002, mais il fonctionnait déjà depuis le mois de janvier 2002. 
les organisations des desocupados, qui donnent priorité aux activités réalisées dans les espaces de travail productif et militant organisés au sein du collectif. Les projets revêtent un caractère social et cherchent à multiplier les ressources pour le fonctionnement des cantines populaires et goûters (potagers, boulangeries, vente de vêtements, etc.).

Si les organisations de desocupados peuvent trouver des marges de manœuvre dans la sélection des bénéficiaires, leur autonomie est limitée. L'implication des organisations telles que les mouvements de sans-emploi comme interlocuteurs lors de l'élaboration en amont des programmes sociaux les concernant leur a permis de peser sur les critères de sélection initialement proposés. Mais cette participation de certaines organisations ne s'est cependant pas traduite par une reconnaissance d'un droit universel au travail pour les bénéficiaires. L'évolution des dispositifs pose la famille, et plus particulièrement les enfants comme destinataires prioritaires de la protection sociale. En ce sens, la revendication des organisations de desocupados d'une «universalisation» des allocations, visant l'accès direct des travailleurs desocupados aux programmes, demeure sans réponse. Cette «universalisation» des politiques sociales reste toutefois un critère de justice sociale porté par les mouvements de sans-emploi, notamment à l'égard des plus jeunes. En effet, avec la réorientation des politiques publiques de lutte contre le chômage en direction des politiques familiales, les jeunes titulaires du programme d'emplois communautaires (PEC) semblent en infraction, les règles d'attribution n'étant pas publiquement affichées. Malgré la dénonciation par ces organisations des restrictions imposées aux jeunes, leur malaise est bien visible tant le risque existe, pour eux, de se voir radiés du programme $^{(47) \text {. }}$

\section{Les PTT et les frontières du social : la remise en cause de la "société salariale"}

L'expansion du travail en Argentine jusqu'au milieu des années 1970 se présentait comme un processus de progrès et d'inclusion sociale qui, à partir des années 1940, avait pris la forme d'une participation des syndicats et des secteurs industriels à travers le Parti péroniste, qui leur conférait une grande capacité de négociation avec l'État et le patronat. Les syndicats participaient aux négociations collectives concernant les conditions de travail (Montes CAtó, 2010) et maîtrisaient une partie du système assurantiel (santé et loisir). Les prestations étaient stratifiées selon le secteur de

(47) Les menaces de dénonciation de la part des voisins ne manquent pas. In fine les jeunes ne sont pas les seuls concernés. l'économie ou les régions. La protection sociale était liée à l'activité salariée, recouvrant les formes corporatives sans les remplacer ${ }^{(48)}$. Ce modèle de société salariale a été défini en Argentine comme étant une «société du travail» caractérisée par des hiérarchies dans le monde du travail, où la situation de chômage n'était pas totalement prise en charge. De ce fait, les organisations de desocupados s'appuient, comme on l'a vu, sur des réseaux d'interconnaissances préexistants qui soulignent les appartenances locales et familiales. Ceux-ci s'avèrent capables d'entretenir des formes de solidarité locale et d'interpeller l'État national sur la question du chômage de masse. Toutefois, les réponses obtenues semblent relever plus d'une logique d'assistance que du droit au travail réclamé par les organisations.

\section{La remise en cause du droit au travail digne : faire "n'importe quel travail" ou s'engager dans une logique de contrepartie}

En dépit de sa formulation sous la forme d'un droit universel, le programme Chefs et cheffes de ménage est désigné comme une politique sociale de sélection des bénéficiaires. Les critères retenus excluent, on l'a vu, une grande partie des anciens allocataires du programme Trabajar et des familles qui, tout en percevant un revenu déclaré, figurent sous le seuil de pauvreté. De même, puisque seul un membre de la famille peut avoir accès au programme, elle ne retient, tout au plus, qu'un des titulaires potentiels qui vivent sous un même toit.

L'existence d'une contrepartie, sous la forme d'une activité de travail, sur le modèle américain du workfare (KRINSKY, 2009), apparaît de manière continue, dans les programmes de première et deuxième génération (49). D'ailleurs, si les organisations de desocupados organisent des manifestations pour rendre effectif l'accès aux droits des travailleurs, ils dénoncent aussi ces contreparties comme relevant de l'exploitation. Au-delà de l'objectif d'extension des quotas, pour répondre à la demande des nouveaux membres, les manifestations cherchent à de fait conférer aux programmes un caractère universel. Mais les réponses obtenues suite à ces revendications se heurtent à la volonté politique. Aucun statut juridique d'ayant droit n'est institué et les acquis de la mobilisation (inclusion des jeunes, treizième mois, capacité de choisir les lieux et les types

(48) À ce sujet, Rubén Lo Vuolo (2010) souligne le caractère «hybride» du système de protection argentin car selon la typologie proposée par Gosta EsPING ANDERSEN (1999), il se rapproche à la fois du type social-démocrate et du type corporatiste.

(49) Contrairement au précédent, le programme Chefs et cheffes de ménage (JJHD) inclut des "composantes», notamment le financement du fonctionnement de projets définis par le ministère du Travail, à hauteur d'au plus $80 \%$ (il ne s'agit pas d'un prêt). 
Tableau 2 : Caractéristiques des programmes de travail selon les gouvernements

\begin{tabular}{|c|c|c|c|}
\hline Gouvernement & $\begin{array}{l}\text { Menem / De la Rua } \\
\text { 1 }^{\text {re génération }}\end{array}$ & $\begin{array}{c}\text { Duhalde / Kirchner } \\
2^{\mathrm{e}} \text { génération }\end{array}$ & $\begin{array}{l}\text { N. Kirchner } \\
3^{\text {e }} \text { génération }\end{array}$ \\
\hline Ministère & \multicolumn{2}{|c|}{ Travail et sécurité sociale } & Développement social \\
\hline Programme & Trabajar & $\begin{array}{c}\text { Chefs et cheffes de ménage } \\
\text { (JJHD) } \\
\text { Programmes d'emploi commu- } \\
\text { nautaires (PEC) }\end{array}$ & $\begin{array}{l}\text { Familles pour l'inclusion } \\
\text { sociale - IDH }\end{array}$ \\
\hline Focalisation & \multicolumn{2}{|c|}{ Poches de pauvreté sur l'ensemble du pays } & Provinces sélectionnées \\
\hline Destinataires & $80 \%$ d'hommes & $70 \%$ de femmes & $\begin{array}{l}\text { Mères ayant trois enfants ou } \\
\text { plus }\end{array}$ \\
\hline Montant & 160 à 200 pesos & 150 pesos & Entre 150 et 225 pesos \\
\hline Contrepartie & $\begin{array}{l}\text { Travail peu qualifié, BTP. } \\
4 \text { à } 6 \text { heures/jour }\end{array}$ & $\begin{array}{c}\text { Travail peu qualifié, fonctionne- } \\
\text { ment des collectivités locales. } \\
4 \text { à } 6 \text { heures/jour }\end{array}$ & $\begin{array}{c}\text { Responsabilité familiale (depuis } \\
2008 \text {, non exigée) }\end{array}$ \\
\hline Durée & 6 mois & $\begin{array}{l}\text { Provisoire. Vaut jusqu'à l'ob- } \\
\text { tention d'un emploi propre ou } \\
\text { du conjoint }\end{array}$ & $\begin{array}{c}\text { Vaut jusqu'à l'obtention d'un } \\
\text { revenu de } 900 \text { pesos par foyer. } \\
\text { Jusqu'à ce que les enfants aient } \\
19 \text { ans }\end{array}$ \\
\hline
\end{tabular}

Tableau établi à partir des données des ministères du Travail (MTSS) et du Développement social (MDS).

d'activités) sont à négocier avec des autorités elles-mêmes instables.

Cette tension entre demande d'accès universel et critique des contreparties exigées traduit le sentiment d'humiliation de ceux qui se voient contraints de «remercier et baisser la tête». Le statut du «travail» productif, bénévole et militant effectué dans le cadre de ces programmes demeure, à bien des égards, problématique. Ces activités sont considérées comme acceptables lorsqu'elles sont comparées à d'autres possibilités qui peuvent servir de repères. C'est surtout le cas dans des familles ayant des aspirations d'ascension sociale et qui investissent fortement dans l'éducation des enfants :

Nora : «Tu trouves ça normal qu'une fille qui a fini ses études vienne manifester dans un piquete?»

Pìa Rius : «Ce serait plus logique qu'elle trouve un travail.»

Nora : «Et que ce soit un travail décent! Parce que pour aller faire le ménage... quand tu fais le ménage, on ne t'utilise pas [comme le feraient certains dirigeants mentionnés plus haut dans l'entretien], ils t'hyperutilisent. Je me rappelle très bien quand j'ai dû le faire. [...] Je ne veux pas que mes filles passent par là.»

(Nora, membre du MTD de Berazategui, août 2005.)

Pour cette mère de famille, la participation de sa fille à un mouvement de travailleurs desocupados prend tout son sens lorsqu'elle est mise en relation avec le peu d'alternatives disponibles. Se faire exploiter par la mairie, se faire «utiliser» par les politiciens ou «hyperutiliser» comme femme de ménage sont autant d'expériences subjectives stigmatisantes qu'elle entend épargner à ses filles. En l'absence de «travail décent» disponible, la mobilisation contestataire, "aller aux piquetes», apparaît soit comme un moindre mal soit comme un «travail pour soi-même».

Si du point de vue du droit, les programmes de travail temporaire ne tiennent pas toutes leurs promesses, dans la perspective de l'action ils apparaissent comme une manière de refuser ce qui est considéré comme indigne. Ils font émerger une critique du travail productif comme seul moyen légitime d'être socialement intégré. Ainsi, bien que l'effort et le temps exigés en contrepartie de l'allocation prévue par le programme soient volontairement sous-rémunérés, ce qui les rapproche des programmes de «workfare» (Morel, 1998; SimONET, 2008), ils ont progressivement accueilli des femmes, comme Nora et sa fille, dont l'engagement devient un rempart contre l'injonction à faire «n'importe quel travail» dans «n'importe quelles conditions».

\section{Universalisation versus sélection : droit au revenu familial}

Pendant la première génération des programmes de travail temporaire, la réponse gouvernementale ne semble pas adaptée à un chômage qui continue de progresser. La deuxième génération se caractérise par l'ampleur de la réponse, en termes quantitatifs (multiplication par douze du nombre d'allocations versées entre 2001 et 2002), et par un déplacement $\mathrm{du}$ registre des politiques conduites, du droit au travail à un droit au revenu familial (cf. tableau 2). Avec les programmes Familles pour l'inclusion sociale (Familias - IDH), la troisième génération a pour finalité, à partir de 2004, d'améliorer l'indice de développement humain (IDH) du Programme des Nations unies pour le développement (PNUD), indice qui remplace la mesure de la pauvreté en 
fonction des seuls revenus ${ }^{(50)}$. Ce dernier programme permet au gouvernement d'instaurer une distinction entre ceux qui seraient les «vrais» desocupados et les «faux»: seuls les premiers restent ciblés par les programmes du ministère du Travail, notamment des programmes de formation; les autres seront, eux, replacés dans des programmes du ministère du Développement social, et sortiront ainsi, de fait, de la population active.

Dans les programmes de travail temporaire, la population évolue et devient principalement féminine à partir de la deuxième génération. Plusieurs éléments expliquent cette tendance : elle est due, d'une part, à l'inclusion des activités sociales (cantines populaires, goûters pour enfants) dans le champ du programme, alors qu'elles en étaient auparavant exclues; d'autre part, la tendance se renforce avec les retours croissants en emploi (formel ou informel) des hommes grâce à la reprise économique de 2003 (les absences et contrats de courte durée déclenchent de fait la sortie du programme).

La troisième génération partage avec les précédentes l'absence de reconnaissance d'un droit universel à un revenu familial. De même, elle donne lieu à une importante mobilisation sociale : si l'augmentation de l'allocation des programmes de travail existants est refusée, les allocations de troisième génération sont d'un niveau plus élevé $\left(225\right.$ pesos $\left.{ }^{(51)}\right)$, soit une augmentation de $50 \%$ par rapport aux programmes précédents. En revanche, et c'est une différence majeure, elles s'inscrivent dans une logique de "désactivation» des personnes, qui sont alors considérées «non employables». Cette évolution se traduit notamment dans la nature des activités réalisées au sein des programmes.

Les mobilisations ayant eu lieu à l'occasion des premiers programmes avaient obtenu que la politique soit reformulée en termes de création d'emplois. La deuxième génération peut, quant à elle, être présentée comme une reconnaissance d'un «droit au revenu minimum», notamment pour les chefs de familles pauvres, hommes comme femmes. Ces présentations sont révélatrices des conditions sociales et politiques qui ont présidé à l'élaboration des programmes et symbolisent les conséquences de la crise : elles sont associées tantôt à «la dissolution» de la société argentine, au chaos

(50) L'indice de développement humain (IDH) est défini selon le Pnud en tenant compte des niveaux de revenu, de l'accès à la santé et à l'éducation. Pour plus d'informations, voir http://hdr.undp.org/fr/content/1\%E2\%80\%99indice-dud\%C3\%A9veloppement-humain-idh; consulté le 10 juillet 2014. (51) En raison de la dévaluation, ce montant est tout de même faible. Le syndicat Central de Trabajadores Argentinos (CTA) réclame un montant minimal de 450 pesos (LozAno, 2003). des émeutes urbaines ${ }^{(52)}$, tantôt à la mise en œuvre d'une politique d'assistanat. La troisième génération des programmes voit le jour dans la suite de cette gestion où l'augmentation du montant des allocations devient l'enjeu d'un bras de fer entre les autorités et les groupes mobilisés. La réponse sera une multiplication des dispositifs en fonction de l'employabilité des titulaires. En contrepartie des allocations, les jeunes doivent suivre une formation et les femmes (dans le cadre du programme Familla $I D H$ ) prendre soin de leurs enfants en bas âge (suivi de carnet de vaccination, présence des enfants à l'école).

Dans ce contexte, la réponse à la crise déclenchée en 2008 s'inscrit d'une manière singulière dans l'histoire des dispositifs sociaux argentins. D'une part, l'État assume sa responsabilité face à la diminution des créations d'emploi due au ralentissement de la croissance économique fin 2008 (le chômage étant à $8,8 \%$ de la population active), et lance des programmes présentés comme «universels», c'est-à-dire accessibles sans conditions de ressources, telle que par exemple l'allocation familiale universelle par enfant (AUH, Asignacion Universal por Hijo, en espagnol). D'autre part, il inaugure de nouveaux programmes «anti-crise», comme le versement des allocations en contrepartie d'un travail, Argentina Trabaja(53). Ce dernier, au sein du ministère du Développement social, semble une nouvelle version des programmes Trabajar, l'activité devant être réalisée cette fois-ci au sein de «coopératives» (créées souvent à cette fin). Comme dans les programmes précédents, des ressources sont attribuées directement aux individus, qui s'engagent à réaliser un travail mais ne sont considérés ni comme des salariés ${ }^{(54)} \mathrm{ni}$ comme des desocupados: ils sont «membres de coopératives», que soutiennent certaines anciennes organisations de desocupados. En revanche les projets à financer semblent s'inscrire dans le cadre plus large des chantiers de travaux publics, engagés par l'État, notamment dans la banlieue de Buenos Aires. Le montant des allocations est très supérieur aux programmes antérieurs, et il est actualisé régulièrement.

Les organisations syndicales de la construction expriment leur désaccord contre ce qu'elles considèrent être des contrats précaires. De leur côté,

(52) Les émeutes urbaines dans les provinces et la ville de Buenos Aires en décembre 2001 sont appelées «estallidos».

(53) Ce dispositif est complété par un programme spécial de diminution des cotisations sociales pour les petites entreprises (Prore).

(54) Au moment de sa création, le montant de l'allocation Argentina Trabaja s'élève à 1200 pesos alors que le salaire minimum est augmenté à 1240 pesos en décembre 2008. L'allocation est ainsi proche du Smic mais ne représente que la moitié du salaire moyen (le salaire moyen dans le privé s'élève à 2400 pesos; CTA, 2008). 
certains mouvements de desocupados ${ }^{(55)}$ dénoncent le caractère clientéliste de ces allocations, notamment pour la sélection des titulaires du programme Argentina Trabaja; ils dénoncent également les procédures de création de coopératives ad hoc. La mobilisation pour l'accès à ces nouveaux programmes de travail est de faible ampleur mais suite à des négociations, elle permet aux organisations de mettre en place de nouveaux projets et d'intégrer de nouveaux membres ${ }^{(56)}$.

D'autres acteurs interviennent également au fur et à mesure que le programme est mis en œuvre : les universités publiques signent des conventions pour délivrer des formations en «gestion coopérative» ainsi que d'autres spécialités techniques (construction, travaux de peinture et bois, etc.), de même que la Confédération générale du travail. À partir de 2010, la réalisation d'une activité en contrepartie de l'allocation peut être remplacée par le suivi d'une formation, visant notamment à achever des études primaires et secondaires afin d'améliorer les conditions d'employabilité de la population active. Comme le signale Jean-Claude BARBIER (2002) à propos des politiques d'activation des chômeurs en Europe, différents éléments de sanction ou de contrôle des chômeurs peuvent être combinés avec des objectifs de solidarité. On peut l'observer ici lorsque la présidente Fernandez de Kirchner considère ces allocations, et notamment l'allocation universelle par enfant, comme un acte de réparation de l'iniquité de traitement entre enfants de salariés et enfants de travailleurs précaires ou de chômeurs (qui étaient auparavant exclus des allocations familiales).

Les familles des titulaires des programmes de travail temporaire de deuxième et troisième génération sont parmi les premiers inscrits au programme AUH (643000), auxquelles de nouvelles familles sont ensuite intégrées (802000 n'ayant pas de protection sociale) ${ }^{(57)}$. Ces accords mettent en exergue la légitimité des enfants comme ayants droit susceptibles de bénéficier de la protection sociale. En revanche, les mères, principales titulaires des AUH, ne sont plus éligibles aux programmes de formation. Emerge ainsi une politique publique d'activation qui cherche à augmenter les conditions d'employabilité des composantes sociales à plus faibles revenus dans un cadre familialiste. Mis en place par des réseaux

(55) Voir notamment, sur http://www.clarin.com/, les articles " Campamento piquetero en la 9 de Julio : acusan al gobierno de "clientelismo" », Clarín digital, 2 novembre 2009 et «Cristina reivindico los planes oficiales de asistencia social», Clarin digital, 21 octobre 2009 ; consultés le 10 juillet 2014. (56) Le nombre de coopératives enregistrées en 2008 est de 12760 , dont $5256-40 \%$ - sont des coopératives de travail enregistrées entre 2003 et 2008 selon l'Inaes (Instituto Nacional de Asociativismo y Economía Social - Institut national de l'économie solidaire).

(57) Elle concerne aussi les enfants des immigrés sur le territoire national depuis plus de trois ans. locaux et associatifs, y compris ceux des collectifs de desocupados, et soumis à de permanentes négociations, seul le revenu familial semble pouvoir s'ériger comme droit des citoyens. Par contre, le versant plus politisé des mobilisations de sansemplois, et notamment les blocages de rues, est désormais stigmatisé et présenté par la présidence de la République comme une «manipulation de la pauvreté ${ }^{(58)} \gg$. Alors même, pourtant, que ces mobilisations rencontrent d'autres conflits qui prennent de l'ampleur, notamment ceux des employés du transport ou de l'enseignement (59).

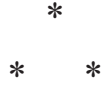

Nous avons rendu compte, à partir de l'expérience de mobilisation des organisations des travailleurs desocupados, des différentes transformations sociales et économiques introduites en Argentine par la généralisation du chômage et des dispositifs mis en place pour y répondre. D’autres conséquences liées à la modification du traitement public du chômage sont apparues : désormais l'intervention de l'État est considérée comme légitime et nécessaire, non seulement comme employeur de dernier recours mais aussi comme rempart contre des formes d'exploitation accrues. Toutefois, les collectifs de sans-emplois n'ont qu'une maîtrise relative des politiques sociales proposées et les accords obtenus s'avèrent fragiles, car ils relèvent de la gestion de crise et sont soumis à des modifications permanentes.

De plus, les modalités d'intervention se transforment au cours du temps, intégrant des propositions émanant des différents acteurs de la scène politique, notamment sous l'influence d'organismes internationaux (Nations unies, Banque mondiale). Différentes contreparties correspondent à des mécanismes d'activation et le développement de ces politiques proches du «workfare» redessinent les frontières de ce qui est un «vrai travail». Cela étant, les effets de la mobilisation de desocupados ne sauraient se mesurer aux seuls acquis de la lutte, les membres qui y ont participé ayant acquis une légitimité pour agir sur d'autres domaines et parfois une dignité pour refuser des emplois aux conditions dégradées.

L'expérience des desocupados montre, malgré (ou du fait de) la crise de la société salariale, que le travail salarié reste un idéal à atteindre. Néanmoins différentes formes de solidarité apparaissent également, comme la revendication d'un travail pour soi, pour sa propre dignité alors que la dimension familialiste de l'intervention sociale,

(58) www.casarosada.gov.ar/discursos/3612/; consulté le 15 juillet 2014.

(59) http://www.clacso.org.ar/institucional/1h.php (OsAL, 2012). 
proche des minima sociaux par son caractère universel, s'inscrit dans la lutte contre la pauvreté et l'engagement au sein des territoires n'est valorisé que comme dernier recours. De ce fait, le revenu minimal d'insertion citoyen ne fut retenu ni par les programmes de deuxième, ni par ceux de troisième génération.

À l'aune de ces constats, l'étude de la gestion de la crise depuis 2001 en Argentine nous invite à remettre en question la manière dont l'activité et le travail sont redéfinis par les politiques publiques de lutte contre le chômage et la pauvreté. Réinterroger la société salariale, c'est se poser la question de la croissance comme objectif prioritaire des États. Avec d'autres, Dominique MéDA (2002) souligne la nécessité d'utiliser des indicateurs plus adaptés, notamment à partir d'une vision patrimoniale du capital social, qui seraient issus d'un débat démocratique.

\section{Bibliographie}

Auyero J. (2002), «Fuego y barricadas. Retratos de la beligerancia popular en la Argentina democrática», Nueva Sociedad (Venezuela), n 179, pp. 144-162.

BAnque mondiale (1997), Informe anual del programa Trabajar, Documento de trabajo.

BARBIER J.-C. (2002), «Peut-on parler d"“activation” de la protection sociale en Europe ?», Revue française de sociologie, vol. 43, $\mathrm{n}^{\circ}$ 2, pp. 307-332.

Beccaria L., Lopez N. (1996), Sin trabajo. Las características del desempleo y sus efectos en la sociedad argentina, Buenos Aires, ed. Losada.

Brusco V., Nazareno M., Stokes S. C. (2005), «Ingreso, región y la calidad de la democracia en la Argentina», in Amaral S., Stokes S. C. (eds.), Democracia local, Buenos Aires, Editorial de la Universidad Nacional de Tres de Febrero.

Castel R. (1995), Les métamorphoses de la question sociale : une chronique du salariat, Paris, Fayard.

CAvarozzi M. (2012), «Modelos de acción presidencial en el siglo XXI latinoamericano», XVII Congreso Internacional del CLAD sobre la Reforma del Estado y de la Administración Pública, Cartagena, Colombia, 30 oct - 2 nov.; disponible en ligne à l'adresse : http://www. dgsc.go.cr/dgsc/documentos/cladxvii/cavarozzi.pdf; consulté le 14 juillet 2014.

Cefaï D. (1996), «La construction des problèmes publics. Définitions de situations dans des arènes publiques », Réseaux, vol. 14, n 75, pp. 43-66.

CEFAÏ D. (2011), «Vers une ethnographie (du) politique : décrire des ordres d'interaction, analyser des situations sociales », in Berger M., Cefaï D., Gayet-Viaud C. (ed.), Du civil au politique. Ethnographies du vivre-ensemble, Bruxelles, Peter Lang, pp. 545-598.

Cels (2001), Informe 2001, Buenos Aires, Eudeba.

Cels (2005), Informe 2004, Buenos Aires, Eudeba.

Collin Delavaud A., Neffa J. C. (dir.) (1994), L'Argentine à l'aube du troisième millénaire, Paris, éditions de l'IHEAL.

CTA (2003), Boletín estadístico del Instituto de estudios y formación.
CTA (2008), Los Hogares Argentinos. Luego de cinco años de crecimiento.

Delich F. (2002), La crisis en la crisis, Buenos Aires, Eudeba.

Di Leo P. (2003), Plan Jefes y Jefas de Hogar Desocupados y régimen social de acumulación neoliberal: una aproximación a sus articulaciones político-simbólicas y estructurales, Instituto de Estudios y Formación de la Central de Trabajadores Argentinos (IDEF/CTA), Buenos Aires.

Demazière D. (2003), Le chômage : comment peut-on être chômeur?, Paris, Belin.

EQuis (2004), Informe sobre pobreza en el Conurbano bonaerense, agosto.

Esping-Andersen G. (1999), Les trois mondes de l'Étatprovidence, Paris, Presses universitaires de France.

Goffman E. (1991), Les cadres de l'expérience, Paris, Éditions Minuit.

Golbert L. (2003), La pobreza Argentina : un problema distributivo, Buenos Aires, Centro Interdisciplinario para el Estudio de las Políticas Públicas, DT n 43.

Golbert L. (2006), « ¿Derecho a la inclusión o paz social? El programa para jefes/as de hogares desocupados», in Franco R., Lanzaro J., Política y políticas públicas en los procesos de reforma latinoamericanos, DF, México, Flacso-México, Cepal, Miño y Davila, pp. 336-360.

GonzÁlez Bombal I. avec Leoni F. (2003), « Sociabilidad en clases medias en descenso: experiencias en el trueque», in Hintze, S. (éd.), Trueque y Economía Solidaria, Buenos Aires, UNGS, pp. 279-310.

Grassi E. (2003), Políticas sociales en la sociedad neoliberal. La otra década infame(I), Buenos Aires, Espacio.

Goulet F., Hernández V. (2011), «Vers un modèle de développement et d'identités professionnelles agricoles globalisés ? Dynamiques d'innovation autour du semis direct en Argentine et en France», Revue Tiers Monde, $\mathrm{n}^{\circ} 207$, pp. 115-132.

Hillcoat G. (1994), «Hétérodoxie et orthodoxie dans les politiques de stabilisation », in Collin Delavaud A., Neffa J. C. (dir.), L'Argentine à l'aube du troisième millénaire, Paris, éditions de l'IHEAL, pp. 99-120. 
INDEC (2007), Mercado de trabajo : principales indicadores.

JANY-CATRICE F., MÉDA D. (2013), «Les nouvelles mesures des performances économiques et du progrès social. Le risque de l'économicisme», Revue du Mauss, vol. $1, \mathrm{n}^{\circ} 41$, pp. 371-397.

Kessler G. (1996), «Alguna implicancias de la experiencia de desocupación para el individuo y su familia», in Beccaria L., Lopez N., Sin trabajo. Las características del desempleo y sus efectos en la sociedad argentina, Buenos Aires, ed. Losada, pp. 111-160.

Kessler G. (2002), La expreriencia educativa fragmentada, Buenos Aires, IIPE.

Kessler G. (2011), «Clairs-obscurs de la structure sociale. Tendances en contrepoint dans l'Argentine du XXI ${ }^{\mathrm{e}}$ siècle», Problèmes d'Amérique latine, $\mathrm{n}^{\circ}$ 82, pp. 93-108.

KlachKo P. (2002), «La conflictividad social en la Argentina de los '90. El caso de las localidades petroleras de Cutral Có y Plaza Huincul, 1996-1997», in Levy B. (coord.), Crisis y conflicto en el capitalismo latinoamericano: lecturas políticas, Buenos Aires, Clacso, pp. 169-221.

KrinSKY J. (2009), «Le workfare. Néolibéralisme et contrats de travail dans le secteur public aux États-Unis », Les notes de l'IES, $\mathrm{n}^{\circ} 8$.

Lazarsfeld P. F., Jahoda M., Zeisel H. (1981), Les chômeurs de Marienthal, Paris, Éditions de Minuit (1 1 re édition 1932).

LE MouËL J. (1981), «Le chômage des jeunes : des vécus très différents », Sociologie du travail, $\mathrm{n}^{\circ} 2$, pp. 163-172.

Lo Vuolo R. (2010), «El Programa “Argentina Trabaja” y el modelo estatico de regulación de la cuestion social en el pais », Buenos Aires, Centro Interdisciplinario para el Estudio de las Políticas Públicas, DT n ${ }^{\circ} 5$.

Lozano C. (2003), Salarios e ingresos en la Argentina contemporánea: El debate sobre la distribución del ingreso, Buenos Aires, CTA.

Margheritis A. (1999), Ajuste y reforma en Argentina 1989-1995 : la economía política de las privatizaciones, Buenos Aires, Nuevo hacer.

Meda D. (2002), «Le capital social. Un point de vue critique », L'Économie politique, ${ }^{\circ} 14$, pp. 36-47.

McGuire J. W. (1996), "Strikes in Argentina: data sources and recent trends ", Latin American research review, vol. 31, n 3, pp. 127-150.

Merklen D. (2005), Pobres ciudadanos, Buenos Aires, Gorla.

Montes Cato J. (2010), «Movimiento obrero y sindicalismo: contradicciones $\mathrm{y}$ fases de acumulación de poder durante los siglos XX y XXI», in Figari C., Lenguita, P., Montes Cato J. (coord.), El movimiento obrero en disputa. La organizacion colectiva de los trabajadores, su lucha y resistencia en la Argentina del siglo XX, Buenos Aires, Ciccus-Ceil Piette Conicet, pp. 41-62.
Morel S. (1998), «Emploi et pauvreté aux États-Unis : les politiques de workfare», in Barbier J.-C., Gautié J. (éd.), Les politiques de l'emploi en Europe et aux ÉtatsUnis, Paris, Presses universitaires de France, pp. 219-234.

NefFa J. C., (2004), «La forma institucional relación salarial y su evolución en la Argentina desde una perspectiva de largo plazo», in Boyer R., Neffa J. C. (coord.), La economia Argentina y su crisis (19762001): visiones institucionalistas y regulacionistas, Buenos Aires, Miño y Davila, CDC, Ceil-Piette Conicet, pp. 105-150.

Neffa J. C., Oliveri M., Persia J., Trucco P. (2010), La crisis de la relación salarial : naturaleza y significado de la informalidad, los trabajos/empleos precarios y los $n^{\circ}$ registrados, Colección Empleo, desempleo \& políticas de empleo, Ceil-Piette Conicet, $\mathrm{n}^{\circ} 1$.

Osal (Observatorio Social de América Latina) (2012), Cronología del Conflicto Social Argentina, diciembre 2012; disponible en ligne à l'adresse : http://www.clacso. org.ar/institucional/1h.php; consulté le 15 juillet 2014.

PAlomino H. (1995), «Quiebres y rupturas de la acción sindical: un panorama desde el presente sobre la evolución del movimiento sindical en la Argentina», in Acuña C. (coord.), La nueva matriz política argentina, Buenos Aires, Nueva Visión, pp. 203-230.

Panigo D. T., ToriJa Zane E. (2004), «Una revisión de las crisis económicas argentinas desde la teoría de la regulación», in Boyer R., Neffa J. C. (coord.), La economia Argentina y su crisis (1976-2001): visiones institucionalistas y regulacionistas, Buenos Aires, Miño y Davila, CDC, Ceil-Piette Conicet, pp. 39-86.

PNud (Programme des Nations unies pour le développement) (2012), Human Developement Report 2012.

Portes A., Hoffman K. (2003), «Las estructuras de clase en América Latina : composicion y cambios durante la época neoliberal », Serie políticas sociales, $n^{\circ} 68$, CepalEclac, Santiago de Chile Mayo.

Quirós J. (2006), Cruzando la Sarmiento. Una etnografía sobre piqueteros en la trama social del sur del Gran Buenos Aires, Buenos Aires, Antropofagia.

Rius P. V. (2007), «Entre travail et activité. Le mouvement des piqueteros en Argentine», Autrepart, $\mathrm{n}^{\circ} 43$, pp. 41-55.

Rius P. V. (2010), Faire valoir sa légitimité. Radicalité et banalité des mouvements de desocupados en Argentine des années 1990 à 2007, thèse de doctorat en sociologie, École des hautes études en sciences sociales.

SANTILlÁN L. (2008), «Prácticas cotidianas e intersecciones entre la Iglesia Católica y grupos familiares en asentamientos populares del Gran Buenos Aires », Cadernos de campo, n 17, pp. 111-132.

Sigal S. (2006), La plaza de Mayo, Buenos Aires, Siglo XXI. 
SChNAPPER D. (1981), L'épreuve du chômage, Paris, Gallimard.

Schutz A. (1987), Le chercheur et le quotidien. Phénoménologie des sciences sociales, Paris, Méridiens-Klincksieck.

Siempro (Sistema de Evaluacion y Monitoreo de Programas Sociales) (2003), Informe de situación laboral.

Siempro (Sistema de Evaluacion y Monitoreo de Programas Sociales) (1998), Programa Trabajar. Características de los beneficiarios.
Simonet M. (2008), «Derrière le voile de la citoyenneté : les usages politiques du volontariat en France et aux États-Unis », Les mondes du travail, $\mathrm{n}^{\circ}$ 5, pp. 53-65.

Svampa M. (ed.) (2000), Desde abajo. La transformación de las identidades sociales, Buenos Aires, UNGS-Biblos.

Svampa M., Pereyra S. (2004), «Les dimensions de l'expérience piquetera: tensions et cadres communs dans l'organisation et la mobilisation des chômeurs en Argentine», Revue Tiers Monde, $\mathrm{n}^{\circ}$ 178, pp. 419-441. 